\title{
May Biodegradable and Biocompatible Polymeric Microneedles be Considered as a Vaccine and Drug Delivery System in the COVID-19 Pandemic?
}

\author{
Biyoparçalanır ve Biyouyumlu Polimerik Mikroiğneler COVID-19 \\ Pandemisinde Aşı ve İlaç Taşıyı ı Sistem Olarak Değerlendirilebilir mi?
}

\author{
(D) Sedat ÜNAL, ID Osman DOĞAN, (D) Yeşim AKTAŞ \\ Erciyes University Faculty of Pharmacy, Department of Pharmaceutical Technology, Kayseri, Turkey
}

Key words: COVID-19, new drug delivery systems, microneedles

Anahtar kelimeler: COVID-19, yeni ilaç taşıyıcı sistemler, mikroiğneler

\section{Dear Editor,}

Coronavirus disease-2019 (COVID-19) was first reported in Wuhan, China at the end of December 2019 and declared as a pandemic. COVID-19 is a disease characterized by acute respiratory failure. COVID-19, which is caused by severe acute respiratory syndrome coronavirus 2 (a member of the Coronaviridae family), has spread to the whole world in a short time due to its rapid transmission from person to person.' New serious mutations giving rise to increasing virus variants and fast spread of the virus have raised questions regarding the efficiacy of vaccines and current treatment methods. Furthermore, supply, stability, and necessity of administering drugs and vaccines developed/being developed for a large number of individuals in a short time brings up a series of problems on a global scale.

Microneedles (MNs) are drug delivery systems that are designed in micron size (usually 10-2000 $\mu \mathrm{m}$ in length and 10$50 \mu \mathrm{m}$ in diameter) and developed mainly for use in transdermal treatments. MNs do not cause pain and discomfort and these can be applied without stimulating the nerve endings by crossing the stratum corneum. Also, MNs can be used without the need for any healthcare personnel and are designed as controlled/ extended systems. Moreover, MNs do not require cold chain for transport. MNs cross the stratum corneum and carry drug molecules to the dermis layer where vascular and lymphatic vessels are concentrated. ${ }^{2}$ Then, drug molecules penetrate lymph or blood capillaries according to their physicochemical properties and enter systemic circulation. MNs, which were first developed in 1976 to overcome obstacles faced with transdermal drug delivery systems, have been designed and scientifically investigated by various research groups as drug delivery systems for various drugs, vaccines, genes, and hormones. On the other hand, parallel to the developments in microfabrication technologies, studies have been performed to increase the applicability of MNs in the pharmaceutical field by obtaining MN types with different designs and characteristics (solid MN, hollow MN, coated MN, and dissolving MN). Intensive scientific studies are being performed by researchers to develop innovative MN systems within the framework of the 
target disease and target molecule to increase potential advantages of $\mathrm{MN}^{3}$ Oral drug administration for children is limited mainly due to the difficulty in swallowing. On the other hand, the parenteral route of drug administration presents difficulties for both children and parents. For injecting drugs, healthcare professionals need to adopt a pedagogical approach. However, this is not enough to eliminate pain and emotional trauma that may occur during drug administration. Therefore, MN designs for children are considered a promising array. Nowadays, MNs are being investigated as an alternative route for efficient and painless insulin delivery for the treatment of type-1 diabetes, which occurs more frequently in childhood. ${ }^{4}$ In another study, promising results were obtained by using ferric pyrophosphate-loaded MNs for treating iron deficiency anemia in children. ${ }^{5}$ In this context, clinical trials are being conducted on MNs developed for children. ${ }^{6}$

In recent years, dissolving MNs have attracted more attention of research groups due to high patient compliance and no residual biological material after application. MN drug delivery systems developed using biocompatible and biodegradable polymers as a basic principle may be used to treat a wide range of diseases. After insertion into the skin, the polymeric matrix forms MN and carrying the drug dissolves and releases the drug molecules. Dissolving MNs also allow modification in release rate and duration according to the type, structure, and molecular weight of the polymers used. ${ }^{7}$ There is no physical wound, incision, or non-biocompatible residual on the skin after the application of dissolving MNs. Promising results have been reported with MN arrays prepared using various biodegradable and biocompatible polymers (such as polylactic acid, hyaluronic acid, poly lactic-coglycolic acid, polyvinyl alcohol PVA, and polyvinylpyrrolidone). ${ }^{8}$ MN systems are also flexible systems that can be modified using advanced studies and multidisciplinary approaches to achieve desired design and drug release rate. ${ }^{3}$

Increasing studies on MN systems in recent years are considered as a harbinger and it is possible that MN systems will be more widely used as drug/vaccine carriers in the near future. It is known that MN vaccination, which was developed especially for influenza immunization and evaluated clinically, is a milestone in this field and has been studied intensively over the last decade. ${ }^{9}$ During the COVID-19 pandemic, the necessity of fast, efficient, stable, easily applicable, and result-oriented systems that do not require compelling storage conditions has again come to the fore. It should not be overlooked that one of the possible global solutions with important advantages in this sense is MN systems.

The COVID-19 pandemic has brought about a large global production and logistics problem in the healthcare field. The production, stability, efficacy, and safety of many sensitive medical materials, such as medicines and vaccines, as well as their rapid and large-scale applications, pose serious difficulties for both healthcare institutions and governments.
Developed vaccine technologies incur more additional costs than drugs. The biggest problem encountered with vaccines today is the lack of healthcare personnels during the use, storage, and transportation of vaccines. Residues such as needles and injectors result in social, environmental, and institutional problems. Therefore, soluble MN systems, which are biodegradable, have the potential to prevent such problems. Being mechanically durable, not requiring any healthcare personnel during application, and being easy to apply without pain are the biggest advantages of MN systems in this field. Studies suggest that MNs can provide controlled/extended release without causing toxicity in the body due to their biocompatible polymeric structure and that it can maintain the immune response for a longer time in vaccine applications.

Biocompatible and biodegradable material-based drug/ vaccine carrier MNs are among the most important candidates in the pharmaceutical and vaccine industry in terms of their advantages, such as logistics, storage, stability, and ease of use. For all these reasons, MN technologies should be further studied and exploited for their use in the treatment of COVID-19. Developments and studies in the field of MNs should be followed closely. Vaccines are important in the prevention of epidemic diseases. Considering the fact that we are facing a pandemic, quick and reliable distribution is of great importance to enable widespread use of vaccines. Based on ongoing studies and acquired knowledge in this field, MN-based soluble COVID-19 vaccines are suggested to be promising.

Conflict of interest: No conflict of interest was declared by the authors. The authors are solely responsible for the content and writing of this paper.

\section{REFERENCES}

1. Yuki K, Fujiogi M, Koutsogiannaki S. COVID-19 pathophysiology: a review. Clin Immunol. 2020;215:108427.

2. Shaikh S, Bhan N, Rodrigues FC, Dathathri E, De S, Thakur G. Microneedle platform for biomedical applications. In: Shaikh S, Bhan N, Rodrigues FC, Dathathri E, De S, Thakur G, eds. Bioelectronics and Medical Devices. London: Elsevier; 2019:421-441.

3. Vora LK, Moffatt K, Tekko IA, Paredes AJ, Volpe-Zanutto F, Mishra D, Peng K, Raj Singh Thakur R, Donnelly RF. Microneedle array systems for long-acting drug delivery. Eur J Pharm Biopharm. 2021;159:44-76.

4. Norman JJ, Brown MR, Raviele NA, Prausnitz MR, Felner El. Faster pharmacokinetics and increased patient acceptance of intradermal insulin delivery using a single hollow microneedle in children and adolescents with type 1 diabetes. Pediatr Diabetes. 2013;14:459-465.

5. Maurya A, Nanjappa SH, Honnavar S, Salwa M, Murthy SN. Rapidly dissolving microneedle patches for transdermal iron replenishment therapy. J Pharm Sci. 2018;107:1642-1647.

6. Duarah S, Sharma M, Wen J. Recent advances in microneedle-based drug delivery: special emphasis on its use in paediatric population. Eur $\mathrm{J}$ Pharm Biopharm. 2019;136:48-69. 
7. van der Maaden K, Jiskoot W, Bouwstra J. Microneedle technologies for (trans)dermal drug and vaccine delivery. J Control Release. 2012;161:645-655.

8. Koyani RD. Synthetic polymers for microneedle synthesis: from then to now. J Drug Deliv Sci Technol. 2020:102071. doi: 10.1016/j. jddst.2020.102071.
9. Frew PM, Paine MB, Rouphael N, Schamel J, Chung Y, Mulligan MJ, Prausnitz MR. Acceptability of an inactivated influenza vaccine delivered by microneedle patch: Results from a phase I clinical trial of safety, reactogenicity, and immunogenicity. Vaccine. 2020;38:7175-7181. 